\title{
Asymmetric heat transport in ion crystals
}

\author{
M. A. Simón $\odot,{ }^{1, *}$ S. Martínez-Garaot, ${ }^{1}$ M. Pons,${ }^{2}$ and J. G. Muga ${ }^{1, \dagger}$ \\ ${ }^{1}$ Departamento de Química-Física, Universidad del País Vasco (UPV/EHU), Bilbao, Spain \\ ${ }^{2}$ Departamento de Física Aplicada I, Universidad del País Vasco (UPV/EHU), Bilbao, Spain
}

(Received 28 May 2019; revised manuscript received 29 July 2019; published 5 September 2019; corrected 3 October 2019)

\begin{abstract}
We numerically demonstrate heat rectification for linear chains of ions in trap lattices with graded trapping frequencies, in contact with thermal baths implemented by optical molasses. To calculate the local temperatures and heat currents we find the stationary state by solving a system of algebraic equations. This approach is much faster than the usual method that integrates the dynamical equations of the system and averages over noise realizations.
\end{abstract}

DOI: 10.1103/PhysRevE.100.032109

\section{INTRODUCTION}

The ideal thermal rectifier, also known as a "thermal diode," is a device that allows heat to propagate in one direction, from a hot to a cold bath, but not in the opposite one when the temperature bias of the baths is reversed. The name is set by analogy to the half-wave rectifiers or diodes for electric current. More generally thermal rectification simply denotes asymmetric heat flows (not necessarily all or nothing) when the bath temperatures are reversed. Thermal rectification was discovered by Starr in 1936 in a junction between copper and cuprous oxide [1]. Many years later, a work of Terraneo et al. demonstrated thermal rectification in a model consisting of a segmented chain of coupled nonlinear oscillators in contact with two thermal baths at temperatures $T_{H}$ and $T_{C}$, with $T_{H}>$ $T_{C}$ [2]. This paper sparked a substantial body of research that spans to this day [3] (see Fig. 1 in Ref. [4]).

Research on thermal rectification has gained significant attention in recent years as a key ingredient in building prospective devices to control heat flows similarly to electrical currents $[4,5]$. There are proposals to engineer thermal logic circuits [6] in which information, stored in thermal memories [7], would be processed in thermal gates [8]. Such thermal gates, as their electronic counterparts, will require thermal diodes and thermal transistors to operate $[9,10]$. Heat-rectifying devices would also be quite useful in nano electronic circuits, letting delicate components dissipate heat while being protected from external heat sources [4].

Most work on thermal diodes has been theoretical with only a few experiments [11-14]. A relevant attempt to build a thermal rectifier was based on a graded structure made of carbon and boron nitride nanotubes that transports heat between a pair of heating and sensing circuits [11]. One of the ends of the nanotube is loaded with a deposition of another material, which makes the heat flow better from the loaded end to the unloaded end. However, rectifications were

\footnotetext{
*miguelangel.simon@ehu.eus

†jg.muga@ehu.es
}

small, with rectification factors (relative heat-flow differentials) around $7 \%$.

Much effort has been aimed at improving the rectification factors and the features of the rectifiers. Some works relied, as in Ref. [2], on a chain segmented into two or more regions with different properties, but using other lattice models such as the Frenkel-Kontorova (FK) model $[15,16]$. The fundamental ingredient for having rectification was attributed to nonlinear forces in the chain [5,15-19], which lead to a temperature dependence of the phonon bands or power spectral densities. The bands may match or mismatch at the interfaces depending on the sign of the temperature bias of the baths, allowing or obstructing heat flow $[2,20]$. Later, alternative mechanisms were proposed which do not necessarily rely on anharmonic potentials [21,22]. Also, Peyrard provided a simple model to explain and build rectifiers based on assuming the Fourier law for heat conduction locally combined with a temperature and position-dependent conductivity [23].

It was soon realized that the performance of segmented rectifiers was very sensitive to the size of the device, i.e., rectification decreases with increasing the length of the rectifier [16]. To overcome this limitation two ideas were proposed. The first one consists in using graded rather than segmented chains, i.e., chains where some physical property varies continuously along the site position such as the mass of particles in the lattice [24-32]. The second one uses particles with long-range interactions (LRI), such that all the particles in the lattice interact with each other $[25,33,34]$. The rationale behind this was that in a graded system new asymmetric, rectifying channels are created, while the long-range interactions create also new transport channels, avoiding the usual decay of heat flow with size [25]. Besides a stronger rectification power, LRI graded chains are expected to have better heat conductivity than segmented ones. This is an important point for technological applications, because devices with high rectification factors are not useful if the currents that flow through them are very small.

In this article we propose to bridge the gap between mathematical models and actual systems exploring the implementation of a heat rectifier in a realistic, graded system 


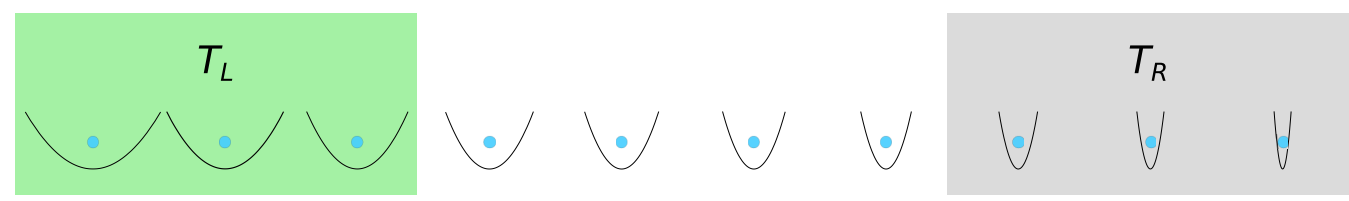

FIG. 1. (Not to scale) Schematic representation of the frequency-graded chain of trapped ions proposed as a thermal rectifier. The left and right ends of the chain are in contact with optical molasses at temperatures $T_{L}$ and $T_{R}$ (green and gray boxes, respectively). Each ion is in an individual trap. The (angular) frequencies of the traps increase homogeneously from left to right, starting from $\omega_{1}$ and ending at $\omega_{1}+\Delta \omega$. The ions interact through the Coulomb force, which is long range, and therefore all the ions interact among them, even distant neighbors. By default we use 15 ions.

with long-range interactions: a chain of ultracold ions in a segmented Paul trap with graded microtraps for each ion. Long-range interactions are due to the Coulomb forces, and the baths at the ends of the chain may be implemented with optical molasses; see Fig. 1. The trapping frequencies of the microtraps are controlled individually in order to create a graded and asymmetric trap-frequency profile along the chain. This asymmetry will lead to a heat flow that depends on the sign of the temperature difference of the baths. Heat transport in trapped ion chains has been studied in several works [35-39], and interesting phenomena like phase transitions have been investigated [35-38]. The idea of using locally controlled traps is already mentioned in Ref. [35] to implement disorder and study its effects. The device we present here may be challenging to implement but in reach with the current technology, in particular that of microfabricated traps [40-42]. Thus the setting is thought for a small, realistic number of controllable ions.

The rest of the article is organized as follows. In Sec. II we describe the physical system of trapped ions with graded trap frequencies. We also set the stochastic dynamics due to the action of lasers at the chain edges. In Sec. III we implement an efficient method to find the steady state using Novikov's theorem and solving an algebraic system of equations. In Sec. IV we present simulations of this system exhibiting thermal rectification and discuss the dependence with ion number, different options for the ion-laser coupling, and the advantages and disadvantages of using a graded frequency profile instead of a segmented one. Finally, in Sec. V we summarize our conclusions and discuss connections with other works.

\section{PHYSICAL SYSTEM}

Consider a linear lattice of $N$ individual harmonic traps of (angular) trapping frequencies $\omega_{n}$ evenly distributed along the $x$ axis at a distance $a$ from each other. Each trap contains a single ion that interacts with the rest via Coulomb potentials. All the ions are of the same species, with mass $m$ and charge $q$. The Hamiltonian that describes the dynamics of the system is (we consider only linear, one-dimensional motion along the chain axis)

$$
H(\boldsymbol{x}, \boldsymbol{p})=\sum_{n=1}^{N}\left[\frac{p_{n}^{2}}{2 m}+\frac{m \omega_{n}^{2}}{2}\left(x_{n}-x_{n}^{(0)}\right)^{2}\right]+V_{\mathrm{int}}(\boldsymbol{x}),
$$

where $\left\{x_{n}, p_{n}\right\}$, the position and momentum of each ion, are the components of the vectors $\boldsymbol{x}, \boldsymbol{p}, x_{n}^{(0)}=n a$ are the centers of the harmonic traps, and $V_{\text {int }}$ is the sum of the Coulomb interaction potential between all pairs of ions,

$$
V_{\text {int }}(\boldsymbol{x})=\frac{1}{2} \sum_{n} \sum_{l \neq n} V_{C}\left(\left|x_{n}-x_{l}\right|\right),
$$

with $V_{C}\left(\left|x_{n}-x_{l}\right|\right)=\frac{q^{2}}{4 \pi \varepsilon_{0}} \frac{1}{\left|x_{n}-x_{l}\right|}$. The ends of the chain are in contact with two thermal reservoirs at temperatures $T_{L}$ for the left bath and $T_{R}$ for the right bath, respectively. The action of the reservoirs on the dynamics of the chain is modeled via Langevin baths at temperatures $T_{L}$ and $T_{R}[43,44]$. The equations of motion of the chain, taking into account the baths and the Hamiltonian, are

$$
\begin{aligned}
& \dot{x}_{n}=\frac{1}{m} p_{n}, \\
& \dot{p}_{n}=-m \omega_{n}^{2}\left(x_{n}-x_{n}^{(0)}\right)-\frac{\partial V_{\text {int }}}{\partial x_{n}}-\frac{\gamma_{n}}{m} p_{n}+\xi_{n}(t),
\end{aligned}
$$

where $\gamma_{n}$ and $\xi_{n}(t)$ are only nonzero for the ions in the end regions, in contact with the left and right baths in the sets $\mathcal{L}=\left\{1,2, \ldots, N_{L}\right\}$ and $\mathcal{R}=\left\{N-\left(N_{R}-1\right), \ldots, N-1, N\right\} ;$ see Fig. 1. Here $\gamma_{n}$ are friction coefficients and $\xi_{n}(t)$ are uncorrelated Gaussian noise forces satisfying $\left\langle\xi_{n}(t)\right\rangle=0$ and $\left\langle\xi_{n}(t) \xi_{m}\left(t^{\prime}\right)\right\rangle=2 D_{n} \delta_{n m} \delta\left(t-t^{\prime}\right), D_{n}$ being the diffusion coefficients. These Gaussian forces are formally the time derivatives of independent Wiener processes (Brownian motions) $\xi_{n}(t)=\sqrt{2 D_{n}} \frac{d W_{n}}{d t}[36,45]$, and Eq. (3) is a stochastic differential equation (SDE) in the Stratonovich sense [45].

The baths are physically implemented by optical molasses consisting of a pair of counterpropagating Doppler-cooling lasers [36]. The friction and diffusion coefficients for the ions in contact with the baths are given by [46]

$$
\begin{aligned}
\gamma_{n} & =-4 \hbar k_{L, R}^{2}\left(\frac{I_{L, R}}{I_{0}}\right) \frac{2 \delta_{L, R} / \Gamma}{\left[1+\left(2 \delta_{L, R} / \Gamma\right)^{2}\right]^{2}}, \\
D_{n} & =\hbar^{2} k_{L, R}^{2}\left(\frac{I_{L, R}}{I_{0}}\right) \frac{\Gamma}{1+\left(2 \delta_{L, R} / \Gamma\right)^{2}}, \\
n & \in \mathcal{L}, \mathcal{R},
\end{aligned}
$$

where $k_{L}\left(k_{R}\right)$ and $I_{L}\left(I_{R}\right)$ are the wave vector and intensity of the left (right) laser. $\delta_{L}\left(\delta_{R}\right)$ is the detuning of the left (right) laser with respect to the angular frequency $\omega_{0}$ of the atomic transition the laser is exciting, and $\Gamma$ is the corresponding natural line width of the excited state. The expressions in Eq. (4) are valid only if the intensities of the lasers are small compared to the saturation intensity $I_{0}, I_{L, R} / I_{0} \ll 1$. In this bath model, the friction term in Eq. (3) comes from the cooling action of the laser, and the white noise force $\xi_{n}(t)$ corresponds to the random recoil of the ions due to spontaneous emission 
of photons [47-49]. Using the diffusion-dissipation relation $D=\gamma k_{B} T$ [50], the temperatures of the optical molasses baths are given by

$$
T_{L, R}=-\frac{\hbar \Gamma}{4 k_{B}} \frac{1+\left(2 \delta_{L, R} / \Gamma\right)^{2}}{\left(2 \delta_{L, R} / \Gamma\right)},
$$

with $k_{B}$ being the Boltzmann constant. If the laser intensities are low enough, the temperatures of the baths are controlled by modifying the detunings. When $\delta=\delta_{D}=-\Gamma / 2$ the optical molasses reach their minimum temperature possible, the Doppler limit $T_{D}=\hbar \Gamma /\left(2 k_{B}\right)$. Note that away from the Doppler limit the same temperature may be achieved for two different values of detuning. These two possibilities imply different couplings (two different pairs of $\gamma$ and $D$ values) and thus different physical effects that will be studied in Sec. IV C.

\section{CALCULATION OF THE STATIONARY HEAT CURRENTS}

The local energy of each site is defined by

$$
H_{n}=\frac{1}{2 m} p_{n}^{2}+\frac{1}{2} m \omega_{n}^{2}\left(x_{n}-x_{n}^{(0)}\right)^{2}+\frac{1}{2} \sum_{l \neq n} V_{C}\left(\left|x_{n}-x_{l}\right|\right) .
$$

Differentiating $H_{n}$ with respect to time we find the continuity equation

$$
\dot{H}_{n}=\frac{p_{n}}{m}\left[\xi_{n}(t)-\gamma_{n} \frac{p_{n}}{m}\right]-\frac{1}{2 m} \sum_{l \neq n} \frac{\partial V_{C}\left(\left|x_{n}-x_{l}\right|\right)}{\partial x_{n}}\left(p_{n}+p_{l}\right) .
$$

Two different contributions can be distinguished: $j_{n}^{B} \equiv$ $\frac{p_{n}}{m}\left[\xi_{n}(t)-\gamma_{n} \frac{p_{n}}{m}\right]$, which is the energy flow from the laser reservoir to the ions at the edges of the chain (only for $n \in \mathcal{L}, \mathcal{R})$, and $\dot{H}_{n}^{i n t} \equiv-\frac{1}{2 m} \sum_{l \neq n} \frac{\partial V_{C}\left(\left|x_{n}-x_{l}\right|\right)}{\partial x_{n}}\left(p_{n}+p_{l}\right)$, which gives the "internal" energy flow due to the interactions with the rest of the ions. In the steady state $\left\langle\dot{H}_{n}\right\rangle=0$, and therefore

$$
\left\langle j_{n}^{B}\right\rangle+\left\langle\dot{H}_{n}^{\text {int }}\right\rangle=0
$$

where $\langle\cdots\rangle$ stands for the expectation value with respect to the ensemble of noise processes $\boldsymbol{\xi}(t)$ ( $\boldsymbol{\xi}$ represents a vector with components $\xi_{n}$ ). Equation (8) implies that, in the steady state, the internal rates $\dot{H}_{n}^{\text {int }}$ vanish for the inner ions of the chain because $j_{n}^{B}=0$ for $n \notin \mathcal{L}, \mathcal{R}$. In chains with nearest-neighbor $(\mathrm{NN})$ interactions, $\left\langle\dot{H}_{n}^{\text {int }}\right\rangle$ simplifies to two compensating and equal-in-magnitude contributions that define the homogeneous heat flux across the chain. For long-range interactions this is not so, and defining the flux is not so straightforward. A formal possibility is to impose $\mathrm{NN}$ interatomic interactions for some atoms in the chain [25], but this approach is not realistic in the current system, so we define instead the heat currents for the left and right baths as

$$
\begin{aligned}
& J_{L}(t)=\sum_{n \in \mathcal{L}}\left\langle j_{n}^{B}\right\rangle, \\
& J_{R}(t)=\sum_{n \in \mathcal{R}}\left\langle j_{n}^{B}\right\rangle,
\end{aligned}
$$

respectively. These expressions are in general time-dependent. In the steady state we must have $J_{L \text {,steady }}+J_{R \text {,steady }}=0$, since the local energies stabilize and internal energy flows cancel. We use either $J_{L \text {,steady }}$ or $J_{R \text {,steady }}$ to calculate the total energy flow in the chain, always taking the absolute value, i.e., $J \equiv$ $\left|J_{L \text {,steady }}\right|=\left|J_{R \text {,steady }}\right| . J$ is defined as $J_{\rightarrow}$ when the hot bath is on the left and $J_{\leftarrow}$ when it is on the right.

To compute the average heat fluxes of the baths $\left\langle j_{n}^{B}\right\rangle$ in Eq. (9) we need the averages $\left\langle p_{n}(t) \xi_{n}(t)\right\rangle$. Instead of explicitly averaging $p_{n}(t) \xi_{n}(t)$ over different realizations of the white noise we use Novikov's theorem $[45,51,52]$. Novikov's theorem states that the ensemble average (over the realizations of the noise) of the product of some functional $\phi(t)$, which depends on a Gaussian noise $\xi(t)$ with zero mean value, $\langle\xi(t)\rangle=0$, and the noise itself is given by

$$
\langle\xi(t) \phi(t)\rangle=\int_{0}^{t} d t^{\prime}\left\langle\xi(t) \xi\left(t^{\prime}\right)\right\rangle\left\langle\frac{\delta \phi(t)}{\delta \xi\left(t^{\prime}\right)}\right\rangle,
$$

where $\delta \phi(t) / \delta \xi\left(t^{\prime}\right)$ is the functional derivative of $\phi(t)$ with respect to the noise, with $t^{\prime}<t$. When the noise is $\delta$-correlated, $\left\langle\xi(t) \xi\left(t^{\prime}\right)\right\rangle=2 D \delta\left(t-t^{\prime}\right)$, and Eq. (10) reads $\langle\xi(t) \phi(t)\rangle=$ $\left.D\left\langle\delta \phi(t) / \delta \xi\left(t^{\prime}\right)\right\rangle\right|_{t^{\prime} \rightarrow t^{-}}$. To apply Novikov's theorem to our model we need the functional derivatives of the position $x_{n}(t)$ and momentum $p_{n}(t)$ coordinates with respect to the white noises. We integrate Eq. (3) to have its formal solution as a functional depending on the white Gaussian noises $\xi_{n}(t)$,

$$
\begin{aligned}
& x_{n}(t)=x_{n}(0)+\frac{1}{m} \int_{0}^{t} d s p_{n}(s), \\
& p_{n}(t)=p_{n}(0)+\int_{0}^{t} d s\left[-\frac{\partial H}{\partial x_{n}}(s)-\frac{\gamma_{n}}{m} p_{n}(s)+\xi_{n}(s)\right] .
\end{aligned}
$$

Equation (11) implies that the functional derivatives are $\delta x_{n}(t) /\left.\delta \xi_{m}\left(t^{\prime}\right)\right|_{t^{\prime} \rightarrow t^{-}}=0$ and $\delta p_{n}(t) /\left.\delta \xi_{m}\left(t^{\prime}\right)\right|_{t^{\prime} \rightarrow t^{-}}=\delta_{n m}\left(\delta_{n m}\right.$ is the usual Kronecker delta symbol). Thus we have $\left\langle x_{n}(t) \xi_{m}(t)\right\rangle=0$ and $\left\langle p_{n}(t) \xi_{m}(t)\right\rangle=\delta_{n m} D_{m}$, which gives for the heat flow from the baths

$$
\left\langle j_{n}^{B}\right\rangle=\frac{1}{m}\left[D_{n}-\gamma_{n} \frac{\left\langle p_{n}^{2}\right\rangle}{m}\right] .
$$

In all simulations we check that $\left|J_{L, \text { steady }}\right|=\left|J_{R \text {,steady }}\right|$ within the numerical tolerance of the computer. To measure the asymmetry of the heat currents we use the rectification factor $R$ defined as

$$
R=\frac{J_{\rightarrow}-J_{\leftarrow}}{\max \left(J_{\rightarrow}, J_{\leftarrow}\right)} .
$$

$R$ values may go from -1 to 1 (in the figures we depict it in percentages between $-100 \%$ and $100 \%$ ). If there is no rectification $J_{\rightarrow}=J_{\leftarrow}$ and $R=0$. For perfect rectification in the right (left) direction, $J_{\rightarrow} \gg J_{\leftarrow}\left(J_{\rightarrow} \ll J_{\leftarrow}\right)$, and $R=1$ $(R=-1)$. Take note that other definitions of rectification factors exist in many works on asymmetric heat transfer, so comparisons should be done with care.

This model does not show the antithermodynamical behavior observed in other models [53,54], and heat is found to flow in all cases from the hot to the cold bath. 


\section{A. Algebraic, small-oscillations approach to calculate the steady state}

To find the temperature profiles and heat currents in the steady state the usual approach is to solve the SDE system in Eq. (3) up to long times and for many realizations of the white noises $\boldsymbol{\xi}(t)$. In that way the ensemble averages $\left\langle p_{n}(t \rightarrow \infty)^{2}\right\rangle$, necessary for both the temperature profiles and heat currents, are computed. This standard route implies a heavy computational effort, in particular when we want to study the heat transport for several bath configurations, frequency increments, and chain parameters. It is possible to circumvent this difficulty and find ensemble averages like $\left\langle x_{n} x_{m}\right\rangle,\left\langle x_{n} p_{m}\right\rangle,\left\langle p_{n} p_{m}\right\rangle$ (second-order moments) without integrating any SDE [55]. The idea is to impose the condition $d\langle\cdots\rangle / d t=0$ for all the second-order moments and linearize the dynamical equations of the system around equilibrium. A system of linear algebraic equations for the moments results, that can be easily solved without solving the SDE many times.

To linearize the SDE in Eq. (3) we approximate the potential energy of the Hamiltonian in Eq. (1), $V(\boldsymbol{x})=$ $V_{\text {int }}(\boldsymbol{x})+m \sum_{n} \omega_{n}^{2}\left(x_{n}-x_{n}^{(0)}\right)^{2} / 2$, by its harmonic approximation around the equilibrium positions $\boldsymbol{x}^{\mathrm{eq}}$, defined by $\left.\frac{\partial V(\boldsymbol{x})}{\partial \boldsymbol{x}}\right|_{\boldsymbol{x}=\boldsymbol{x}^{\mathrm{eq}}}=0$. The approximate potential (ignoring the zeropoint energy) is

$$
V(\boldsymbol{x}) \approx \frac{1}{2} \sum_{n, m} K_{n m}\left(x_{n}-x_{n}^{\mathrm{eq}}\right)\left(x_{m}-x_{m}^{\mathrm{eq}}\right),
$$

with $K_{n m}=\left.\frac{\partial^{2} V(x)}{\partial x_{n} \partial x_{m}}\right|_{\boldsymbol{x}=\boldsymbol{x}^{\text {eq }}}$ being the Hessian matrix entries of $V(\boldsymbol{x})$ around the equilibrium configuration [56]

$$
K_{n m}=\left\{\begin{array}{ll}
m \omega_{n}^{2}+2\left(\frac{q^{2}}{4 \pi \varepsilon_{0}}\right) \sum_{l \neq n} \frac{1}{\left|x_{n}^{\mathrm{eq}}-x_{l}^{\mathrm{eq}}\right|^{3}} & \text { if } n=m \\
-2\left(\frac{q^{2}}{4 \pi \varepsilon_{0}}\right) \frac{1}{\left|x_{n}^{\mathrm{eq}}-x_{m}^{\mathrm{eq}}\right|^{3}} & \text { if } n \neq m
\end{array} .\right.
$$

Note that this approximation does not modify the two main features of the system, namely, asymmetry and long-range interactions, which are manifest in the asymmetric distribution of $\omega_{n}$ and the nonzero off-diagonal elements of the $K$ matrix, respectively. In the following we will use $y_{n}=x_{n}-x_{n}^{\mathrm{eq}}$ to simplify the notation. The linearized dynamics around the equilibrium positions are given by

$$
\begin{aligned}
& \dot{y}_{n}=\frac{1}{m} p_{n}, \\
& \dot{p}_{n}=-\sum_{l} K_{n l} y_{l}-\frac{\gamma_{n}}{m} p_{n}+\xi_{n}(t) .
\end{aligned}
$$

Now we set $d\langle\cdots\rangle / d t=0$ for all the moments. Using Eq. (16) and applying Novikov's theorem we find

$$
\begin{aligned}
\left\langle p_{n} p_{l}\right\rangle-\gamma_{l}\left\langle y_{n} p_{l}\right\rangle-\sum_{m} K_{l m}\left\langle y_{n} y_{m}\right\rangle & =0, \\
\sum_{m}\left[K_{n m}\left\langle y_{m} p_{l}\right\rangle+K_{l m}\left\langle y_{m} p_{n}\right\rangle\right]+\frac{1}{m}\left(\gamma_{l}+\gamma_{n}\right)\left\langle p_{n} p_{l}\right\rangle & =2 \delta_{n l} D_{n} .
\end{aligned}
$$

The system (17) is linear in the second-order moments so it can be solved numerically to find the steady-state values of the moments. Besides Eq. (17) we have that $\left\langle y_{n} p_{l}\right\rangle=-\left\langle y_{l} p_{n}\right\rangle$, which follows from Eq. (16) and $d\left\langle y_{n} y_{m}\right\rangle / d t=0$. Since there are $\frac{1}{2} N(N-1)$ independent $\left\langle y_{n} p_{l}\right\rangle$ moments, we choose the ones with $n<l$. Similarly, the moments $\left\langle y_{n} y_{l}\right\rangle$ and $\left\langle p_{n} p_{l}\right\rangle$ contribute with $\frac{1}{2} N(N+1)$ independent variables each, and we choose the ones with $n \leqslant m$. Thus there are in total $\frac{1}{2} N(3 N+1)$ independent moments that we arrange in the vector

$$
\begin{aligned}
\eta= & {\left[\left\langle y_{1} y_{1}\right\rangle,\left\langle y_{1} y_{2}\right\rangle, \ldots,\left\langle y_{N} y_{N}\right\rangle,\right.} \\
& \times\left\langle p_{1} p_{1}\right\rangle,\left\langle p_{1} p_{2}\right\rangle, \ldots,\left\langle p_{N} p_{N}\right\rangle, \\
& \left.\times\left\langle y_{1} p_{2}\right\rangle,\left\langle y_{1} p_{3}\right\rangle, \ldots,\left\langle y_{N-1} p_{N}\right\rangle\right]^{T} .
\end{aligned}
$$

There are the same number of independent equations as independent moments: $N^{2}$ equations correspond to the first line in Eq. (17), and $\frac{1}{2} N(N+1)$ equations to the second line because of the symmetry with respect to $n, l$. The system of equations (17) may be compactly written as $\mathbf{A} \boldsymbol{\eta}=\mathbf{B}$, where $\mathbf{A}$ and $\mathbf{B}$ are a $\frac{1}{2} N(3 N+1)$ square matrix and vector.

\section{NUMERICAL RESULTS}

We now display the results of our simulations. To find the temperature profiles and the currents in the steady state we use the algebraic method described in Sec. III A. We also check that the results coincide with those by solving Eq. (3) for many different realizations of the noisy forces $\boldsymbol{\xi}(t)$ and averaging. The code for all the numerical simulations has been written in the language JULIA [57,58]. In particular, to solve the Langevin equation, we used the Julia package DIFFERENTIALEQUATIONS.JL [59].

To model the baths and the chain we use atomic data taken from ion trap experiments [60,61]. We consider $15{ }^{24} \mathrm{Mg}^{+}$ ions in all figures except in Fig. 6. The three leftmost and three rightmost ions are illuminated by Doppler cooling lasers. The Doppler cooling lasers excite the transition $3 s^{2} S_{1 / 2} \rightarrow$ $3 p^{2} P_{1 / 2}$, with angular frequency $\omega_{0}=2 \pi \times 1069 \mathrm{THz}$ and excited state line width $\Gamma=2 \pi \times 41.3 \mathrm{MHz}$ [36]. For this ionic species and atomic transition the Doppler limit is $T_{D}=$ $1 \mathrm{mK}$. The intensities of the laser beams are small compared to the saturation intensity $I_{0}$ so that Eq. (4) holds. We take $I_{n} / I_{0}=0.08$ for the ions in the laser beams, whereas $I_{n}=0$ for the rest.

The temperatures $T_{L}, T_{R}$ of the left and right laser baths are controlled with their detunings $\delta_{L}, \delta_{R}$ with respect to the atomic transition. We fix two values for the detunings, $\delta_{H}$ and $\delta_{C}$, such that $T_{H}>T_{C}$ (hot and cold baths, also source and drain), and we define $J_{\rightarrow}\left(J_{\leftarrow}\right)$ as the stationary heat current in the chain when $T_{L}=T_{H}$ and $T_{R}=T_{C}\left(T_{L}=T_{C}\right.$ and $\left.T_{R}=T_{H}\right)$.

Except in Sec. IV E we consider a graded frequency profile. If the frequency of the leftmost trap is $\omega_{1}$, the frequency of the $n$th trap will be $\omega_{n}=\omega_{1}+\Delta \omega \frac{n-1}{N-1}$ up to $\omega_{1}+\Delta \omega$ for the rightmost trap. In Sec. IV E we compare the graded chain to a segmented chain, where the left half of the chain has trapping frequencies $\omega_{1}$ and the other half has $\omega_{1}+\Delta \omega$.

\section{A. Evolution to steady state}

To compare the results by solving Eq. (3) and averaging and those from the algebraic method we simulated a frequency-graded chain with a trapping frequency 

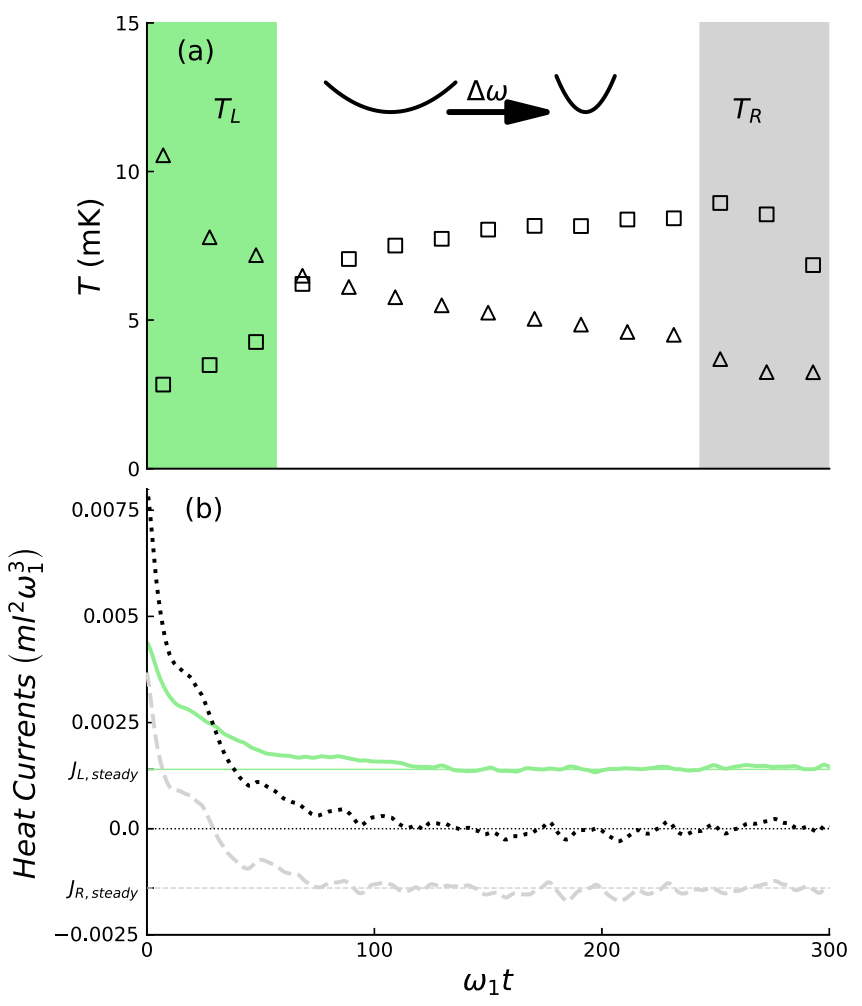

FIG. 2. (a) Temperatures of the ions in the stationary state for a graded chain with the parameters described in Sec. IV A. The temperature profiles found with the algebraic method [Eq. (17)] are indistinguishable from the ones found solving the Langevin equation [Eq. (3)]. Empty triangles (squares) correspond to $T_{L}=T_{H}\left(T_{L}=\right.$ $\left.T_{C}\right)$ and $T_{R}=T_{C}\left(T_{R}=T_{H}\right)$. (b) Heat currents as a function of time for $T_{L}=T_{H}$ and $T_{R}=T_{C}$ [see Eq. (9)]: $J_{L}(t)$ (thick solid green line) from the left reservoir into the chain; $J_{R}(t)$ (thick dashed grey line) from the right reservoir into the chain (negative except at very short times); $J_{L}(t)+J_{R}(t)$ (thick dotted black line), which must go to zero in the steady state. The three lines tend to stationary values marked by the horizontal thin lines. Parameters: $\omega_{1}=2 \pi \times 50 \mathrm{kHz}$, $a=50 \mu \mathrm{m}, \delta_{H}=-0.02 \Gamma$, and $\delta_{C}=-0.1 \Gamma$, which gives temperatures $T_{H} \approx 12 \mathrm{mK}$ and $T_{C} \approx 3 \mathrm{mK}$. $\Delta \omega=0.5 \omega_{1}$. In all figures $\Gamma=2 \pi \times 41.3 \mathrm{MHz}$.

$\omega_{1}=2 \pi \times 50 \mathrm{kHz}$ for the leftmost ion; see Fig. 2. The number of ions interacting with the laser beams (three on each bath) is consistent with the lattice constant and typical waists of Gaussian laser beams [60,61]. To set the trap distance we fix first the characteristic length $l=\left(\frac{q^{2}}{4 \pi \varepsilon_{0}} \frac{1}{m \omega_{1}^{2}}\right)^{1 / 3}$ as the distance for which the Coulomb repulsion of two ions equals the trap potential energy for an ion at a distance $l$ away from the center of its trap. If $a<l$, the Coulomb repulsion of the ions is stronger than the trap confinement which makes the ions jump from their traps. With the parameters used in this section we have $l=38.7 \mu \mathrm{m}$ and set $a=1.29 l=50 \mu \mathrm{m}$. The detunings of the hot and cold lasers are $\delta_{H}=-0.02 \Gamma$ and $\delta_{C}=-0.1 \Gamma$, which gives temperatures $T_{H} \approx 12 \mathrm{mK}$ and $T_{C} \approx 3 \mathrm{mK}$. We fix the value $\Delta \omega=0.5 \omega_{1}$ for the frequency increment.

The results of the two methods are in very good agreement. In the scale of Fig. 2(a) the calculated local temperatures
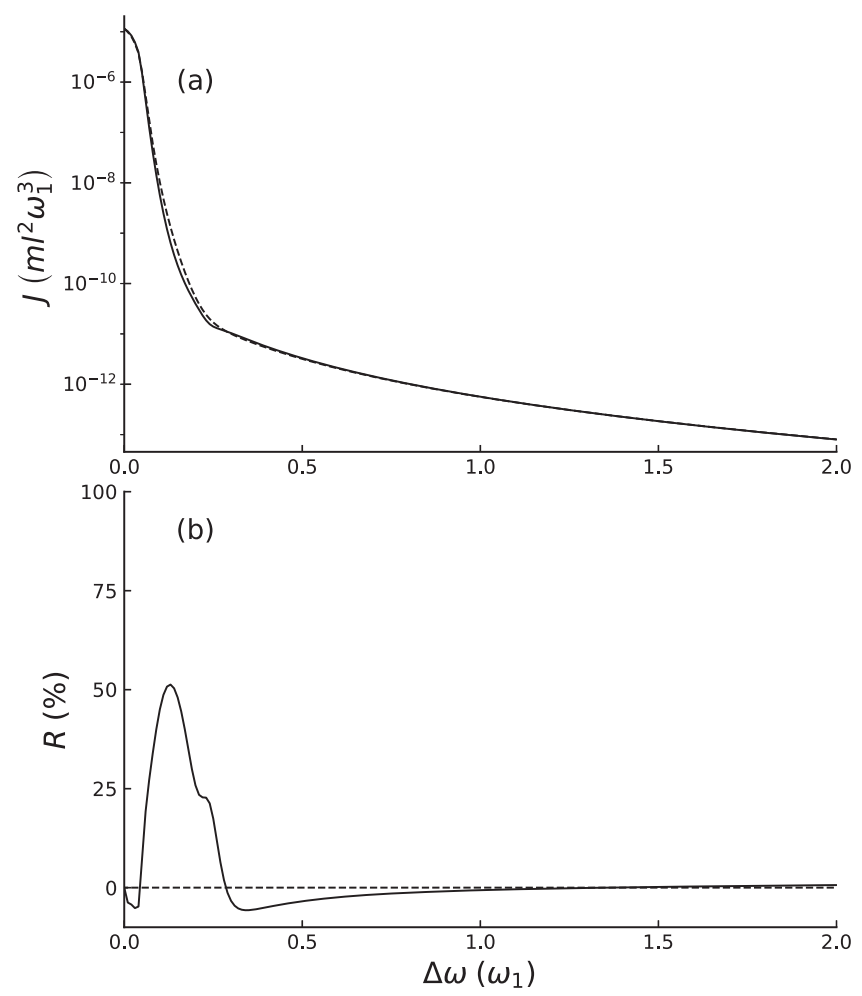

FIG. 3. Graded chain of $N=15^{24} \mathrm{Mg}^{+}$ions. (a) Stationary fluxes for different frequency increments: $J_{\rightarrow}$ (for $T_{L}=T_{H}$ and $T_{R}=$ $T_{C}$, dashed line); $J_{\leftarrow}$ (for $T_{L}=T_{C}$ and $T_{R}=T_{H}$, solid line). (b) Rectification factor. Parameters: $\omega_{1}=2 \pi \times 1 \mathrm{MHz}, l=5.25 \mu \mathrm{m}, a=$ $4.76 l(25 \mu \mathrm{m}), \delta_{H}=-0.02 \Gamma$, and $\delta_{C}=-0.1 \Gamma$.

are undistinguishable. In the calculation based on solving the dynamics we had to integrate Eq. (3) for $N_{\text {trials }}=1000$ realizations of white noise $\boldsymbol{\xi}(t)$. The method based on the system of moments shortened the calculation time with respect to the dynamical trajectories by a factor of $1 / 700$. In fact, the time gain is even more important because the dynamical method requires further processing, performing a time averaging to compute the stationary flux in addition to noise averaging; see Fig. 2(b).

Additionally, the relaxation to the steady state slows down when the frequencies of the traps increase since the deterministic part of the Langevin equation dominates the dynamics over the stochastic part, entering an underdamped regime. In contrast, this increase does not affect the algebraic method.

\section{B. Rectification in frequency-graded chains}

In this subsection we demonstrate rectification for the frequency-graded chain. We used the method described in Sec. III A for ${ }^{24} \mathrm{Mg}^{+}$ions with the same parameters for the baths used before. We fix the trapping frequency of the leftmost trap to $\omega_{1}=2 \pi \times 1 \mathrm{MHz}$ and a trap spacing $a=4.76 l$ $(25 \mu \mathrm{m}$ ) (the characteristic length is $l=5.25 \mu \mathrm{m}$ ). Figure 3 depicts the results with these parameters in a graded chain. Figure 3(a) shows that both $J_{\rightarrow}$ and $J_{\leftarrow}$ decrease rapidly as the frequency increment is increased. The rectification reaches its maximum value for a frequency difference of $\Delta \omega \approx 0.1 \omega_{1}$. The fluxes cross so there are some points where 


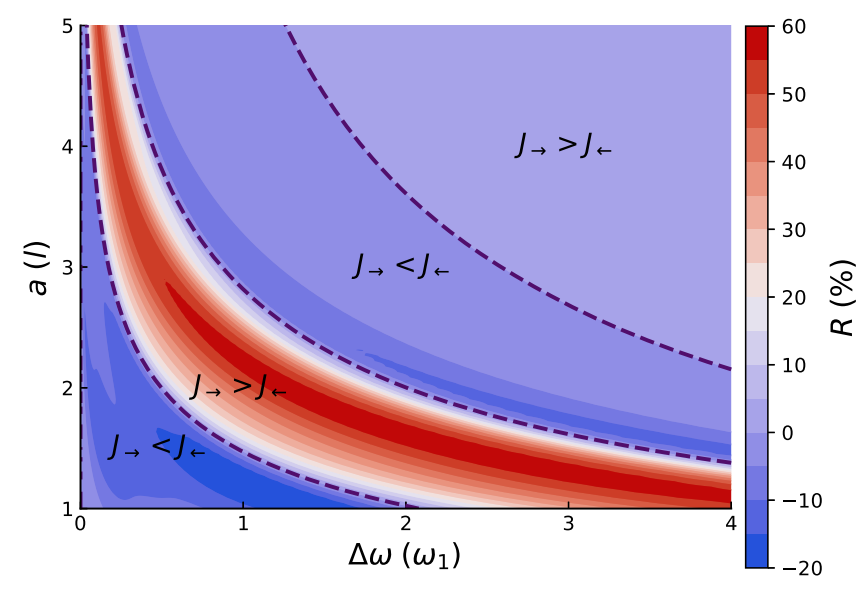

FIG. 4. Rectification factor in a graded chain of $N=15^{24} \mathrm{Mg}^{+}$ ions for different trap distances and frequency increment. The dashed lines are for $R=0$ and delimit the regions $J_{\rightarrow}>J_{\leftarrow}$ and $J_{\rightarrow}<J_{\leftarrow}$. The parameters are $\omega_{1}=2 \pi \times 1 \mathrm{MHz}, l=5.25 \mu \mathrm{m}, \delta_{H}=-0.02 \Gamma$, and $\delta_{C}=-0.1 \Gamma$.

the rectification is exactly zero, besides the trivial one at $\Delta \omega=0$, at $\Delta \omega=0.05 \omega_{1}, 0.3 \omega_{1}, 1.3 \omega_{1}$. At these points the direction of rectification reverses, presumably as a consequence of the changes in the match and mismatch of the temperature-dependent local power spectra. The change of rectification direction occurs for all choices of parameters, as displayed in Fig. 4. Figure 4 gives the rectification factor for different trap distances and frequency increments. Zero-rectification curves separate regions with different rectification direction. The second region in Fig. 4 (starting from the left) would be the most interesting one to build a thermal diode, since rectification reaches its largest values there.

For small values of $\Delta \omega$ there is little asymmetry in the chain and therefore modest rectification is expected, whereas a very large $\Delta \omega$ implies very high trapping frequencies on the right implying too strong a confinement and vanishing interactions. This bottleneck decreases the fluxes in both directions and the rectification. However, since $\Delta \omega$ is controllable, and the range of values of $\Delta \omega$ for which rectification is larger can be also controlled with the intertrap distance $a$ (see Fig. 4), the existence of a rectification window does not imply a major limitation.

\section{Same bath temperatures, different bath couplings}

As already mentioned below Eq. (5), above and below the detuning $\delta_{D}=-\Gamma / 2$ corresponding to the Doppler limit temperature, the optical molasses allow for two different couplings [two pairs of friction and diffusion coefficients in Eq. (4)] between the ions and the laser corresponding to the same bath temperature. This duality may be seen explicitly in Fig. 5. Specifically Fig. 5(a) depicts the variation of the friction coefficient for values of $\delta$ around $\delta_{R}$, and Fig. 5(b) the corresponding temperatures. Interestingly, the different couplings imply different rectification factors. If we set $\delta_{C}=$ $\delta_{D}=-\Gamma / 2$, i.e., the cold bath is cooled to the Doppler limit, $\delta_{H}$ can be chosen to be below or above $\delta_{D}$ for the same temperature $T_{H}$. The corresponding rectification factors for
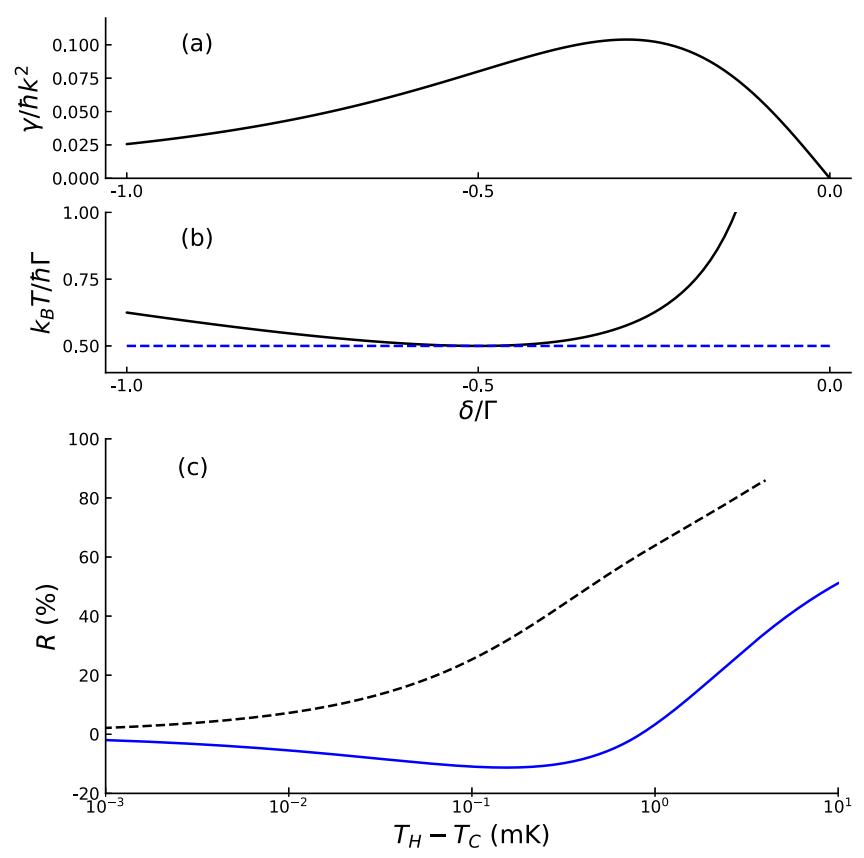

FIG. 5. (a) Friction coefficient defined in Eq. (4). (b) Bath temperature defined in Eq. (5). (c) Rectification as a function of the temperature difference between the hot and cold baths $T_{H}-T_{C}$ for $\delta_{H}$ below (dashed black line) and above (solid blue line) the Doppler limit, and $\delta_{C}=\delta_{D}$ (Doppler limit). Parameters: $\omega_{1}=2 \pi \times 1 \mathrm{MHz}$, $\Delta \omega=0.15 \omega_{1}, l=5.25 \mu \mathrm{m}, a=4.76 l$.

the two choices are shown in Fig. 5(c), which demonstrates that significant rectification can be achieved by choosing $\delta_{H}<\delta_{D}$ for temperature increments that are smaller than or of the order of $T_{C}=T_{D}$, for example, $R \approx 20 \%$ for $\Delta T=$ $0.1 T_{C}$, or $R \approx 60 \%$ for $\Delta T=T_{C}$. Finding good rectification at low (relative) temperature differences is considered to be one of the challenges in asymmetric heat transport research [62].

\section{Dependence with ion number}

Keeping in mind that scaling the frequency-graded ion chain to a large numbers of ions is not a realistic option in this setting, it is nevertheless important to study the dependence with ion number from small to moderate numbers. In Fig. 6 we observe an overall trend in which the rectification decreases with the number of ions in the chain (while it increases with temperature bias $\Delta T$ in the studied range). This effect is easy to understand, as increasing $N$ while keeping the total variation of the trapping frequency $\Delta \omega$ constant, the frequency gradient decreases. This lowers the asymmetry in the chain and the rectification factor. Oscillations with $N$ superimposed to the global trend are more visible at the smaller $N$ values giving an optimal $N$ value at $N=19$.

\section{E. Graded versus segmented}

We have also compared the performance of the graded thermal diode and a segmented version in which the left half of the chain is trapped with frequency $\omega_{1}$ and the right half (including the middle ion) with $\omega_{1}+\Delta \omega$. Even though the 


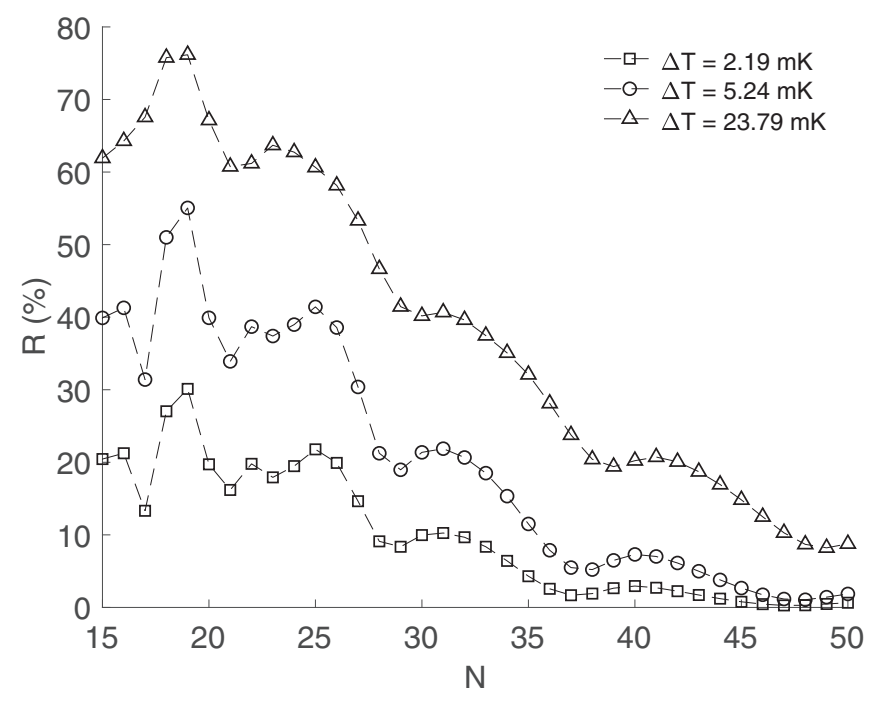

FIG. 6. Rectification factor for different bath temperature differences $\Delta T$ as the number of ions is increased. The detuning of the cold bath laser is set to the Doppler limit $\delta_{C}=-\Gamma / 2 . \omega_{1}=$ $2 \pi \times 1 \mathrm{MHz}, \Delta \omega=0.15 \omega_{1}, l=5.25 \mu \mathrm{m}, a=4.76 l$.

optimal rectification in Fig. 7(a) for the segmented chain is larger than for the graded chain, the fact that the fluxes are generally much larger for the graded chain [see Fig. 7(b)] makes the graded chain more interesting for applications.
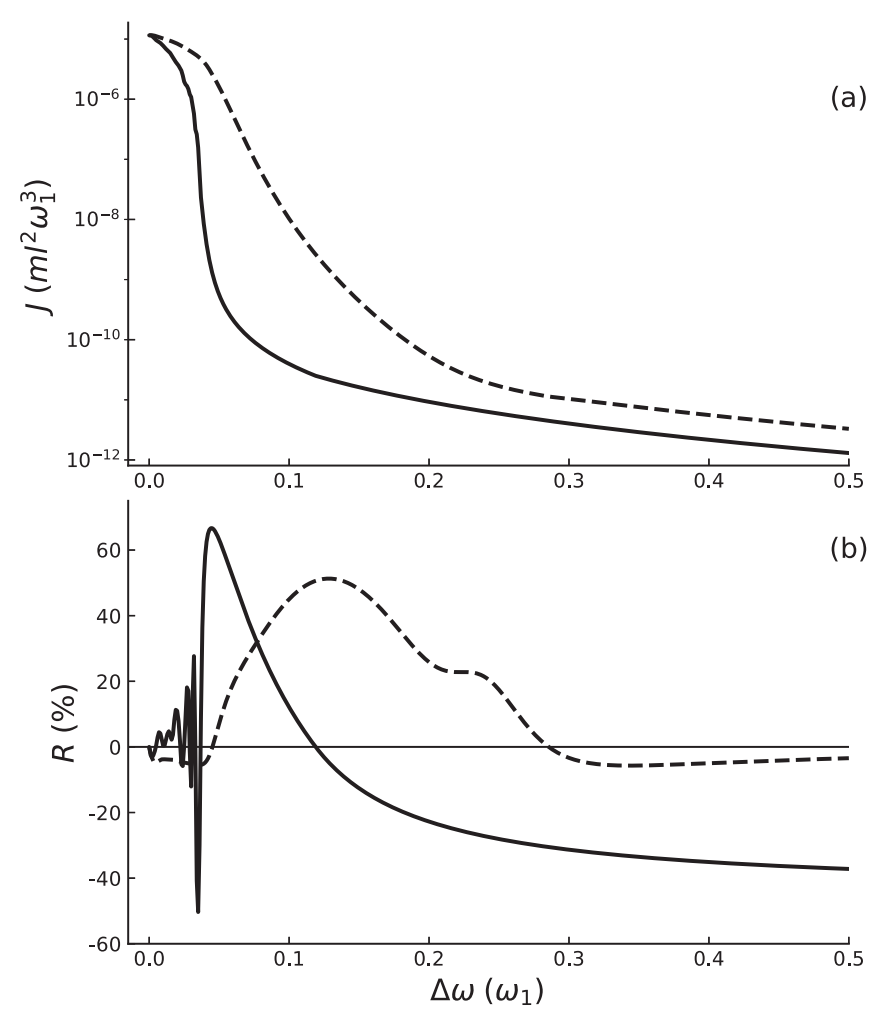

FIG. 7. Comparison of graded and segmented chains with $N=$ $15^{24} \mathrm{Mg}^{+}$ions. (a) Maximum of $J_{\rightarrow}$ and $J_{\leftarrow}$ for the graded and segmented chain for different frequency increments. (b) Rectification factor: graded chain (dashed lines); segmented chain (solid lines). Parameters: $\omega_{1}=2 \pi \times 1 \mathrm{MHz}, l=5.25 \mu \mathrm{m}, a=$ $4.76 l, \delta_{H}=-0.02 \Gamma$, and $\delta_{C}=-0.1 \Gamma$.

\section{SUMMARY AND DISCUSSION}

In this article we have numerically demonstrated heat rectification in a chain of ions trapped in individual microtraps with graded frequencies, connected at both ends to thermal baths created by optical molasses. An alternative to implement a graded frequency profile in the laboratory could be combining a collective Paul trap for all the ions with on-site dipolar laser forces [35,63-65].

A goal of this article is to connect two communities, ion trappers and researchers on heat-rectification models. The results found are encouraging and demonstrate the potential of a trapped-ion platform to experimentally investigate heat rectification schemes. Trapped ions are quite interesting to this end because they are highly controllable and may easily adopt several features to enhance rectification, such as the ones explored here (long-range interactions and an asymmetrical gradation), or others such as time-dependent forces [5,66], or different nonlinearities in on-site forces. The limitations and application domain should also be clear; the proposed platform is circumscribed to cold temperatures of the order of hundreds of $\mu \mathrm{K}$ to $\mathrm{mK}$ achieved by Doppler cooling. In this sense it is not aimed at competing with (it is rather complementary to) proposals for which experiments [11-14] or simulations $[62,67,68]$ demonstrate thermal rectification at room temperature or for hundreds of $\mathrm{K}$. Also, the number of ions should realistically be kept small so the proposed ion chain is not aimed at achieving a macroscopic diode length, but at playing a role in thermal diode research and in the context of ion-trapped based quantum technologies.

Methodologically, the calculation of the steady state has been performed with an algebraic approach much faster than the time-consuming integration and averaging over noise and time of the dynamical equations. The algebraic approach linearizes the forces around equilibrium positions, which, in this system and for the realistic parameters considered, is well justified and tested numerically. The results found provide additional evidence that simple linear models may rectify heat flow [21]. We underline that our linear model is, arguably, even simpler than some linear "minimalist, toy models" in Ref. [21] that showed rectification (our on-site forces are already linear from the start, and the temperature dependence of explicit model parameters is only in the coefficients of the Langevin baths), with the important bonus of being also realistic.

To shed some more light on the mechanism behind the observed rectification we can analyze the local thermal conductivities $\lambda[x, T(x)]$ defined in a continuous model by [23]

$$
J=\lambda[x, T(x)]\left|\frac{d T(x)}{d x}\right|,
$$

where $J$ is the stationary heat current and $T(x)$ the local temperature. [We use the modulus of the temperature derivative for consistency with our (positive) definition of $J$.] In our model we discretize the coordinate with the ion index and the temperature derivative is discretized as

$$
\frac{d T_{n}}{d x}=\frac{T_{n+1}-T_{n-1}}{x_{n+1}^{\mathrm{eq}}-x_{n-1}^{\mathrm{eq}}} .
$$




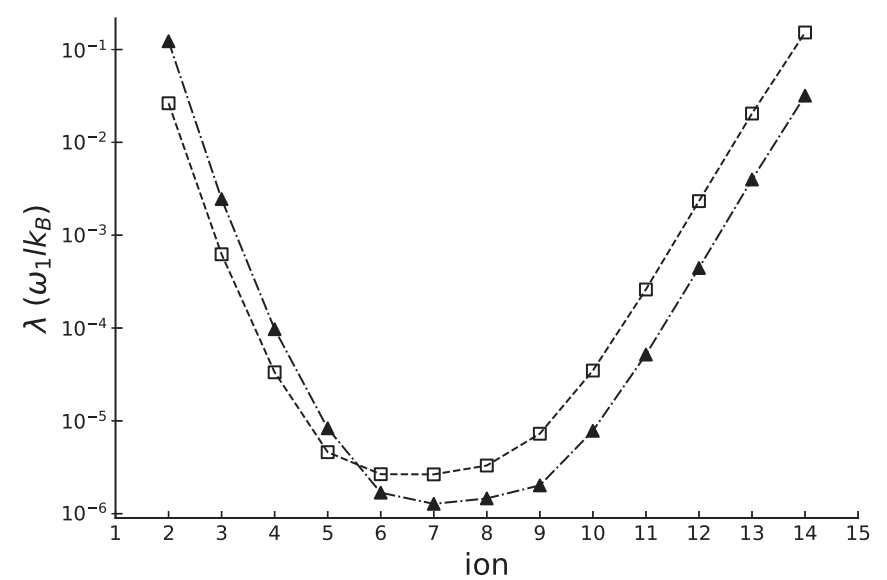

FIG. 8. Thermal conductivity through the chain for $T_{L}>T_{R}$ (empty squares), and $T_{L}<T_{R}$ (filled triangles). $\omega_{1}=2 \pi \times 1 \mathrm{MHz}$, $\Delta \omega=0.15 \omega_{1}, l=5.25 \mu \mathrm{m}, a=4.76 l, \delta_{H}=-0.02 \Gamma$, and $\delta_{C}=$ $-0.1 \Gamma$.

Through integration, it is clear that when $\lambda$ depends on both temperature and position, rectification is possible. In the continuous model the temperature increment between the baths is

$$
\left|T_{L}-T_{R}\right|=\int_{0}^{L} \frac{J}{\lambda[x, T(x)]} d x
$$

so that the key for rectification is a different integral of the inverse of the conductivities in the two scenarios $\left(T_{L}=\right.$ $T_{H}, T_{R}=T_{C}$ with conductivity $\lambda_{\rightarrow}\left[x, T_{\rightarrow}(x)\right]$ along a local temperature decreasing from the left or the reversed one, $T_{R}=T_{H}, T_{L}=T_{C}$ with conductivity $\lambda_{\leftarrow}\left[x, T_{\leftarrow}(x)\right]$ along an increasing local temperature. Particularly favorable for rectification is the scenario where one of the lambdas is above the other one for all $x$. Figure 8 shows that this is essentially the case in our model, at least along the most relevant part of the integral.

\section{ACKNOWLEDGMENTS}

We thank Daniel Alonso, Joseba Alonso, Gabriele de Chiara, and Emmanuel Pereira for fruitful discussions and comments. M.A.S. acknowledges support by the Basque Government predoctoral program (Grant No. PRE-2018-2-0177). We acknowledge funding from Grants No. PGC2018-101355B-I00 (MCIU/AEI/FEDER,UE) and Fis2016-80681-P and from the Basque Government (Grant No. IT986-16).
[1] C. Starr, Physics 7, 15 (1936).

[2] M. Terraneo, M. Peyrard, and G. Casati, Phys. Rev. Lett. 88, 094302 (2002).

[3] E. Pereira, Europhys. Lett. 126, 14001 (2019).

[4] N. Roberts and D. Walker, Int. J. Thermal Sci. 50, 648 (2011).

[5] N. Li, J. Ren, L. Wang, G. Zhang, P. Hänggi, and B. Li, Rev. Mod. Phys. 84, 1045 (2012).

[6] Z.-Q. Ye and B.-Y. Cao, Nanoscale 9, 11480 (2017).

[7] L. Wang and B. Li, Phys. Rev. Lett. 101, 267203 (2008).

[8] L. Wang and B. Li, Phys. Rev. Lett. 99, 177208 (2007).

[9] B. Li, L. Wang, and G. Casati, Appl. Phys. Lett. 88, 143501 (2006).

[10] K. Joulain, J. Drevillon, Y. Ezzahri, and J. Ordonez-Miranda, Phys. Rev. Lett. 116, 200601 (2016).

[11] C. W. Chang, D. Okawa, A. Majumdar, and A. Zettl, Science 314, 1121 (2006).

[12] W. Kobayashi, Y. Teraoka, and I. Terasaki, Appl. Phys. Lett. 95, 171905 (2009)

[13] D. M. Leitner, J. Phys. Chem. B 117, 12820 (2013).

[14] M. Elzouka and S. Ndao, Sci. Rep. 7, 44901 (2017).

[15] N. Li, P. Hänggi, and B. Li, Europhys. Lett. 84, 40009 (2008).

[16] B. Hu, L. Yang, and Y. Zhang, Phys. Rev. Lett. 97, 124302 (2006).

[17] N. Zeng and J.-S. Wang, Phys. Rev. B 78, 024305 (2008).

[18] G. Katz and R. Kosloff, Entropy 18, 186 (2016).

[19] G. Benenti, G. Casati, C. Mejía-Monasterio, and M. Peyrard, From thermal rectifiers to thermoelectric devices, in Thermal Transport in Low Dimensions: From Statistical Physics to Nanoscale Heat Transfer, edited by S. Lepri (Springer International, Cham, 2016), pp. 365-407.

[20] B. Li, L. Wang, and G. Casati, Phys. Rev. Lett. 93, 184301 (2004).
[21] E. Pereira, Phys. Rev. E 96, 012114 (2017).

[22] M. Pons, Y. Y. Cui, A. Ruschhaupt, M. A. Simón, and J. G. Muga, Europhys. Lett. 119, 64001 (2017).

[23] M. Peyrard, Europhys. Lett. 76, 49 (2006).

[24] J. Wang, E. Pereira, and G. Casati, Phys. Rev. E 86, 010101(R) (2012).

[25] S. Chen, E. Pereira, and G. Casati, Europhys. Lett. 111, 30004 (2015).

[26] M. Romero-Bastida, J. O. Miranda-Peña, and J. M. López, Phys. Rev. E 95, 032146 (2017).

[27] N. Yang, N. Li, L. Wang, and B. Li, Phys. Rev. B 76, 020301(R) (2007).

[28] M. Romero-Bastida and J. M. Arizmendi-Carvajal, J. Phys. A 46, 115006 (2013).

[29] R. Dettori, C. Melis, R. Rurali, and L. Colombo, J. Appl. Phys. 119, 215102 (2016).

[30] E. Pereira, Phys. Rev. E 82, 040101(R) (2010).

[31] E. Pereira, Phys. Rev. E 83, 031106 (2011).

[32] R. R. Ávila and E. Pereira, J. Phys. A 46, 055002 (2013).

[33] D. Bagchi, Phys. Rev. E 95, 032102 (2017).

[34] E. Pereira and R. R. Ávila, Phys. Rev. E 88, 032139 (2013).

[35] N. Freitas, E. A. Martinez, and J. P. Paz, Phys. Scr. 91, 013007 (2016).

[36] A. Ruiz, D. Alonso, M. B. Plenio, and A. del Campo, Phys. Rev. B 89, 214305 (2014).

[37] A. Ruiz-García, J. J. Fernández, and D. Alonso, Phys. Rev. E 99, 062105 (2019).

[38] T. Pruttivarasin, M. Ramm, I. Talukdar, A. Kreuter, and H. Häffner, New J. Phys. 13, 075012 (2011).

[39] M. Ramm, T. Pruttivarasin, and H. Häffner, New J. Phys. 16, 063062 (2014).

[40] J. I. Cirac and P. Zoller, Nature (London) 404, 579 (2000). 
[41] F. Krauth, J. Alonso, and J. Home, J. Phys. B 48, 015001 (2014).

[42] R. Schmied, J. H. Wesenberg, and D. Leibfried, Phys. Rev. Lett. 102, 233002 (2009).

[43] S. Lepri, R. Livi, and A. Politi, Phys. Rep. 377, 1 (2003).

[44] A. Dhar, Adv. Phys. 57, 457 (2008).

[45] R. Toral and P. Colet, Stochastic Numerical Methods: An Introduction for Students and Scientists (John Wiley \& Sons, New York, 2014).

[46] W. D. Phillips, Laser cooling and trapping of neutral atoms, in Laser Manipulation of Atoms and Ions, edited by E. Arimondo, W. D. Phillips, and F. Strumia (Elsevier Science, Amsterdam, 1993), pp. 289-344.

[47] H. J. Metcalf and P. Van der Straten, Laser Cooling and Trapping, Graduate Texts in Contemporary Physics (SpringerVerlag, New York, 1999).

[48] H. J. Metcalf and P. van der Straten, J. Opt. Soc. Am. B 20, 887 (2003).

[49] C. Cohen-Tannoudji, Atomic motion in laser light, in Fundamental Systems in Quantum Optics, edited by J. Dalibard, J. M. Raimond, and J. Zinn-Justin (North-Holland, Amsterdam, 1993).

[50] L. Chee Kong, arXiv:1008.0491 (2010).

[51] E. A. Novikov, Sov. J. Exp. Theor. Phys. 20, 1290 (1965).

[52] P.-W. Ma and S. L. Dudarev, Phys. Rev. B 83, 134418 (2011).

[53] G. D. Chiara, G. Landi, A. Hewgill, B. Reid, A. Ferraro, A. J. Roncaglia, and M. Antezza, New J. Phys. 20, 113024 (2018).

[54] A. Levy and R. Kosloff, Europhys. Lett. 107, 20004 (2014).
[55] S. Särkkä and A. Solin, Applied Stochastic Differential Equations, Institute of Mathematical Statistics Textbooks (Cambridge University Press, Cambridge, 2019).

[56] D. F. James, Appl. Phys. B 66, 181 (1998).

[57] J. Bezanson, S. Karpinski, V. B. Shah, and A. Edelman, arXiv:1209.5145 (2012).

[58] J. Bezanson, A. Edelman, S. Karpinski, and V. Shah, SIAM Rev. 59, 65 (2017).

[59] C. Rackauckas and Q. Nie, Discrete Contin. Dyn. Syst. B 22, 2731 (2017).

[60] F. M. Leupold, Bang-bang control of a trapped-ion oscillator, Ph.D. thesis, ETH Zurich, 2015.

[61] H.-Y. Lo, Creation of squeezed Schrödinger's cat states in a mixed-species ion trap, Ph.D. thesis, ETH Zurich, 2015.

[62] T. Zhang and T. Luo, Small 11, 4657 (2015).

[63] M. Enderlein, T. Huber, C. Schneider, and T. Schaetz, Phys. Rev. Lett. 109, 233004 (2012).

[64] A. Bermudez, M. Bruderer, and M. B. Plenio, Phys. Rev. Lett. 111, 040601 (2013).

[65] C. Schneider, M. Enderlein, T. Huber, and T. Schaetz, Nat. Photonics 4, 772 (2010).

[66] A. Riera-Campeny, M. Mehboudi, M. Pons, and A. Sanpera, Phys. Rev. E 99, 032126 (2019).

[67] H. Ma and Z. Tian, Nano Lett. 18, 43 (2018).

[68] K. M. Reid, H. D. Pandey, and D. M. Leitner, J. Phys. Chem. C 123, 6256 (2019).

Correction: A typographical error introduced during the production process has been fixed in Eq. (4). 